\title{
ARHYTHMORHYNCHUS ANSER N. SP., NOUVEL ACANTHOCÉPHALE PARASITE DE LARUS ARGENTATUS CACHINNANS (PONTOP.) SUR LE LITTORAL ROUMAIN DE LA MER NOIRE
}

\author{
Par Bujor FLORESCU
}

Pendant nos recherches de parasitologie, entreprises en juillet et août $\mathrm{I}_{93}{ }_{7}$ sur les poissons et oiseaux du littoral roumain de la Mer Noire, à la Station de Zoologie maritime d'Agigea (Constantza), nous avons disséqué de nombreux exemplaires du genre Larus, dont $2 \mathrm{I}$ exemplaires de l'espèce Larus argentatus cachinnans (Pontop.). Dans l'intestin de deux de ces oiseaux nous avons trouvé, une fois 3 et l'autre fois 16 exemplaires adultes, mâles et femelles, d'un Acanthocéphale ayant les caractéristiques du genre Arhythmorhynchus Lühe, mais différant de toutes les descriptions d'espèces connues, par des caractères tranchés.

La liste des espèces déjà décrites, appartenant au genre Arhythmorhynchus dans le monde entier est, selon nos connaissances actuelles, la suivante :
I. A. brevis Van Cleave.
2. A. duocinctus Chandler.
3. A. frassoni Molin.
4. A. fuscus Harada.
5. A. hispidus Van Cleave (1).
6. A. invaginabilis v. Linstow.
7. A. longicollis Villot.
8. A. macrourus Bremser.
9. A. plicatus v. Linstow.
ro. A. pumilirostris Van Cleave.

(I) Witenberg (1932) crée, pour l'espèce Arhythmorhynchus hispidus de Van Cleave, un nouveau genre, Southwellina. Mais Chandler (r935) considère comme prématurée la tendance de Witenberg à diviser le genre Arhythmorhynchus en plusieurs genres, les connaissances actuelles sur ces vers étant encore insuffisantes pour de tels démembrements.

Ansales de Parastrologie, T. XVIII, Nos 4-5-6. - 1941, p. 215-219. 
11. A. roseus Molin (larva).

12. A. siluricola Dollfus (larva).

13. A. teres Van Cleave.

14. A. trichocephalus R. Leuckart.

I5. A. uncinatus Kaiser.

De ces 15 espèces d'Arhythmorhynchus, 3 seulement ont été trouvées chez des hôtes rapprochés du nòtre, c'est-à-dire: $A$. frassoni chez Larus marinus (Italie, Europe), A. longicollis chez Larus canus et Larus ridibindus (Bretagne, Spitzberg, Angleterre) et A. teres chez Larus dominicanus (Talcahuano, Chili).

Nous donnons la description de notre nouvelle espèce :

Arhythmorhynchus anser n. sp.

Grande espèce, de la taille d'Arhythmorhynchus frassoni, rencontrée assez rarement dans la dernière moitié de l'intestin de Larus argentatus cachinnans (Pontop), sur le littoral roumain de la Mer Noire; le rapport du parasitisme (exemplaires parasités : exemplaires étudiés) est 2 : 2 r. Nos exemplaires ont été récoltés en juillet et. août. La taille des exemplaires mâles et femelles n'est pas appréciablement différente, comme pour les autres espèces d'Acanthocéphales. Le corps est très long et filiforme, sa couleur générale sur le vif est jaunàtre. Nos exemplaires étaient fortement accrochés à la muqueuse de l'intestin des hòtes.

Corps. - Fortement allongé, ayant un élargissement ovoïde, semblable au renflement qu'on rencontre chez Arhythmorynchus frassoni, présent chez les deux sexes et renfermant les testicules chez les mâles. La partie du corps proprement dite, antérieure au renflement dont nous venons de parler, est couverte dans sa portion antérieure par des épines disposées en rangées régulières qui avancent plus bas sur le côté ventral de l'animal, sans arriver pourtant, comme c'est le cas pour Arhythmorhynchus frassoni, jusqu'au renflement même. Dans le reste, la largeur du corps est à peu près constante, décroissant imperceptiblement vers la fin.

Les femelles adultes ont une longueur totale variant de $6 \mathrm{I}$ à $70 \mathrm{~mm}$. avec un diamètre moyen de $1 \mathrm{~mm}$. 33. La largeur maxima au niveau du renflement antérieur du corps est, pour l'exemplaire figuré, de I mm. 83.

Les mâles sont longs de 44 à $63 \mathrm{~mm}$.; la largeur au niveau du ren- 
flement antérieur du corps, mesurée sur l'exemplaire figuré, est de I mm. 55, la largeur moyenne pour le mème exemplaire étant de I $\mathrm{mm}$. го.

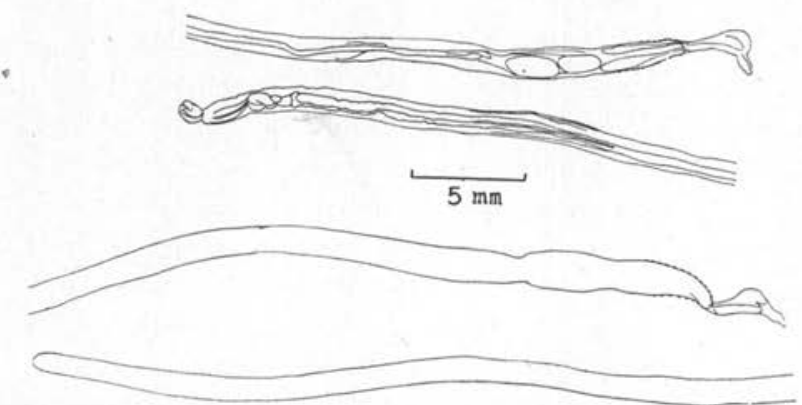

Fig. I. - Arhythmorhynchus anser n. sp.

Cou. - Très long et portant le caractère spécifique de cette nouvelle espèce, s'élargissant dans sa portion distale par rapport au corps proprement dit dans une bosse accentuée dorsalement. La trompe sort

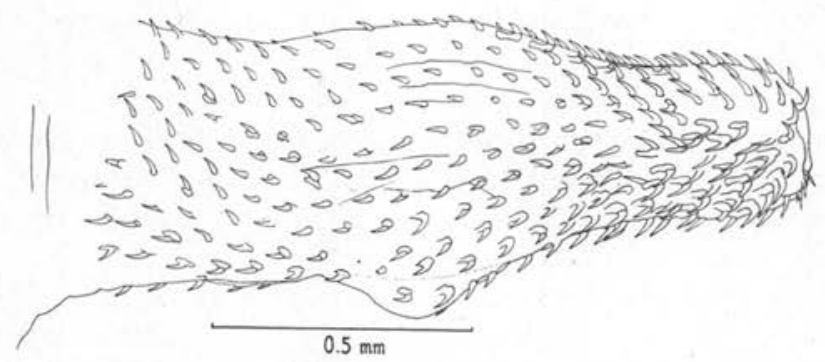

Fig. 2. - Arhythmorhynchus anser, trompe.

de ce renflement globulaire dans une direction plus ou moins ventrale. Le cou portant ainsi la trompe donne à l'animal entier l'aspect d'une canne à tête d'oie, fait qui nous a suggéré le nom d'anser que nous donnons à cette nouvelle espèce.

Les dimensions du cou pour les deux exemplaires mâle et femelle sont les suivantes :

Male : longueur du cou $3 \mathrm{~mm}$. 3o, largeur minima en bas du cou, 
o mm. 4o, largeur maxima au niveau du renflement globulaire postproboscidien, I $\mathrm{mm}$. I 5 .

Femelle : longueur du cou, $3 \mathrm{~mm}$. 46, largeur minima (mesurée comme pour le mâle), o mm. 5o, largeur maxima, r mm. i3.

Trompe. - De forme allongée-fusiforme, l'extrémité distale ayant l'aspect d'un cône tronqué ; largeur maxima au milieu de sa longueur. Dimensions pour les deux exemplaires figurés :

Male : trompe incomplètement évaginée; longueur totale, $950 \mu$; largeur maxima au milieu, $480 \mu$; largeur

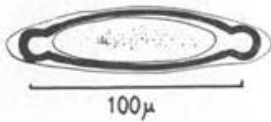

Fig. 3. - OEuf d'Arhythmorhynchus an. ser. minima à la base, $430 \mu$; largeur minima pour la partie antérieure, $200 \mu$.

Femelle : longueur totale de la trompe complètement évaginée, I mm. 46; largeur maxima au milieu, $500 \mu$; largeur minima à la base, $470 \mu$; largeur minima pour la partie antérieure, $280 \mu$.

22 rangées longitudinales de 22 crochets chacune; tous les crochets d'une même rangée, de dimensions sensiblement égales. Pas de crochets plus grands au milieu des rangées ventrales.

Réceplacle de la trompe. Male : $4 \mathrm{~mm} .67$ de long, o mm. 22 de large. Femelle : $4 \mathrm{~mm}$. o4 de long, o mm. 37 largeur maxima.

Lemnisques. - Longues de $4 \mathrm{~mm}$. à $4 \mathrm{~mm}$. 20, largeur maxima, $0 \mathrm{~mm}$. 5 o à o mm. 67 .

Testicules. - Ils commencent à $4 \mathrm{~mm}$. du pli basal du cou : l'antérieur est long de $2 \mathrm{~mm}$. et large de o $\mathrm{mm}$. 85, le postérieur est long de $2 \mathrm{~mm}$. 45 et large de a mm. 95. La bourse copulatrice évaginée est longue de $2 \mathrm{~mm}$. $\mathrm{r} 5$, large de o $\mathrm{mm}$. 55 .

Longueur totale des deux glandes prostatiques, $37 \mathrm{~mm} .86$.

Cufs. - En moyenne : longueur r $23 \mu$, largeur $33 \mu$, renflements globulaires bien développés sur la deuxième enveloppe.

Exemplaires types: $\mathrm{N}^{\circ}{ }_{1} 233$, mâle et femelle, dans la collection de l'auteur. Cotypes, Nos I 224 et I $233 a$, dans la même collection et $1233 b$, dans la collection scientifique du Musée Régional de Bessarabie, Chisinau, Roumanie.

Hote : Larus argentatus cachinnans (Pontop.).

Localisation : la dernière moitié de l'intestin.

Localité : Agigea (district de Constanza), Roumanie. 


\section{Bibliograpilie}

Chander (A. C.). - Parasites of fishes of Galveston Bay. Proc. U. S. National Museum, LXXXIII, r $935, \mathrm{n}^{\circ} 2977$, pp. $15 \mathrm{r}-\mathrm{r} 57$, pl. XII, fig. 5-9.

Harada (I.). - Ueber eine neue Species der Aeanthocephalen. Japan. Jl. Zool., II, 1929 , pp. $195-198$, pl. VI.

MeYer (A.). - Acanthocephala, 2 vol., in : $\mathrm{D}^{r} \mathrm{H}$. G. Bronn's Klassen und Ordnungen des Tierreichs, 4, 2 Sect., 2, pp. 1-582, 383 fig. et une planche, 1932. Acanthocephala, in : Brohmer, Die Tierwelt Mitteleuropas, I, 6, p 40, 46 fig., 1938

Ravther (M.). - Acanthocephala = Kratzwürmer, in : Handbuch der Zoologie, von Th. Krumbach, II, I, p. 449 (4)-482 (4), fig. 48r-523, 1929-193o.

Van Cleave (H. J.). - Acanthocephala collected by the Swedish Expedition to the Juan Fernandez Islands (1916-1917). The Natural History of the Juan Fernandez Istand, IH, I920, pp. 75-8o, pl. 5 .

A revision of the genus Arhythmorhynchus with description of two new species from North American birds. $J l$. of Parasitology, II, r9x6, pp. 167$174,2 \mathrm{pl}$.

Witenberg (G.). - Akanthocephalen-Studien. I. Ueber einige für die Systematik der Akanthocephalen wichtige anatomische Merkmale. Boll. di zool., pubbł. dall'Unione Zoologica Italiana, III, r932, pp. 2/43-252, 5 fig.

Akanthocephalen-Studien. II. Ueber das System der Akanthocephalen. Boll. di zool., pubbl. dall'Unione Zoologica Italiana, III, 1932, pp. 253-265, 3 fig.

Station de zoologie maritime d'Agigea (Constanza) et Musée régional de Bessarabie, Chisinau, Roumanie. 